\title{
PRÉVENTION DE LA BIOCORROSION DES ACIERS INOXYDABLES par modification de la composition chimique
}

\author{
C. Lemaitre $(*)$, G. Hernandez $(*)$, G. Béranger $(*)$ \\ et J. Guezennec $(* *)$ \\ (*) Division "matériaux", dépt. G. M., Université de Compiègne \\ (**) Ifremer, centre de Brest, Département Environnement Profond
}

By using electrochemical measurements and bacteria counting, we have verified that the added elements in an AISI $316 \mathrm{~L}$ stainless steel, as poison allowing elements for the bacteria, like copper or arsenic, change the electrochemical properties versus the time, and modifie the structure of the bacterial population on the surface. Comparisons of the obtained results in synthetic sea water, sterilized or inoculated, and in natural sea water have permitted to observe very great differences versus the added elements. The use of sterilized natural sea water and then inoculated, gives results which permit to show the specific role of the aerobic and the anaerobic bacteria.

Les phénomènes rencontrés lors de la corrosion des aciers inoxydables en milieu chloruré conduisent généralement à l'existence de piqûres, dont le processus de formation peut être combattu par une modification de la composition chimique de l'acier, en y introduisant par exemple du molybdène. En milieu marin, la présence de micro-organismes, et notamment de bactéries, peut rendre l'alliage plus sensible à la corrosion localisée [1]. D'un point de vue électrochimique, on peut observer un décalage des potentiels d'équilibre tendant à favoriser la formation des piqûres; on peut se demander si d'autres éléments d'addition pourraient contrecarrer ce type de dégradation. Pour cela, on peut envisager d'ajouter, au cours de l'élaboration de la nuance, des éléments chimiques susceptibles d'être toxiques vis-à-vis des bactéries réputées pour favoriser la corrosion.

Les micro-organismes présents dans le milieu ont tendance à former un voile biologique qui s'établit à l'interface solide-liquide $[2,3]$. De plus, à la surface de l'acier inoxydable un film de passivité se forme, en équilibre avec le milieu environnant $[4,5]$. Lorsque le voile biologique s'installe, il le fait aux dépends de ce film passif. Celui-ci peut donc être localement rompu, et l'alliage devient alors sensible à la corrosion. Ceci peut être amplifié par la présence des bactéries elles-mêmes, lorsqu'elles modifient, à cause de leur métabolisme, le milieu environnant : certaines d'entre elles produisent par exemple des composés soufrés, qui deviennent très corrosifs dans les conditions locales rencontrées à l'interface $[6,7]$. Le rôle des éléments toxiques ajoutés serait ainsi de prévenir la formation du voile biologique, et donc d'augmenter la stabilité du film passif vis-à-vis de ce type d'agression.

C'est dans ce cadre que nous avons entrepris, sur un acier inoxydable austénitique du type AISI $316 \mathrm{~L}$, une étude des modifications du comportement à la corrosion induites par 
l'addition, dans sa composition chimique, d'éléments toxiques pour les bactéries qui sont susceptibles de s'accrocher à la surface du métal, et d'amorcer ainsi la formation du voile biologique.

\section{Conditions expérimentales}

Nous avons choisi, pour effectuer nos mesures, trois nuances d'acier : AISI $316 \mathrm{~L}$, AISI $316 \mathrm{~L}+\mathrm{Cu}$, AISI $316 \mathrm{~L}+$ As. Les deux dernières nuances ont été obtenues par la refusion de la première avec adjonction des éléments poison à l'état liquide $\left({ }^{*}\right)$. L'état de référence est celui d'une hypertrempe classique : $1100^{\circ} \mathrm{C}, 15 \mathrm{mn}$, refroidissement à l'eau. L'état de surface de nos échantillons est celui obtenu après un polissage au carbure de silicium de granulométrie décroissante jusqu'à une grosseur de $800 \mu \mathrm{m}$.

L'eau de mer synthétique a été fabriquée au laboratoire selon les indications de l'ASTM. Nos mesures dans ce milieu ont été réalisées à la température ambiante, en présence d'une solution agitée, avec une aération naturelle. Dans ce milieu, nous avons pour certains cas de figure introduit, avec un milieu de culture, des souches bactériennes prélevées sur des échantillons métalliques naturellement corrodés en eau de mer, souches que nous n'avons pas déterminées précisément.

L'eau de mer naturelle provient de Brest, où les mesures correspondantes ont été réalisées. Pompée et filtrée, elle est distribuée dans les laboratoires d'Ifremer, à une température voisine de $15^{\circ} \mathrm{C}$, avec un $\mathrm{pH}$ de 8 à 8,2 . Cette eau a pu, pour certaines études, être stérilisée $\left(120^{\circ} \mathrm{C}\right.$ sous 1 bar pendant $\left.20 \mathrm{mn}\right)$. On ajoutait alors de la levure comme source alimentaire $\left(10 \mathrm{mg} . \mathrm{l}^{-1}\right)$, afin de préparer une inoculation ultérieure.

Les techniques d'étude que nous avons mises en œuvre sont des mesures électrochimiques quasi-stationnaires (évolution dans le temps du potentiel libre, courbes de polarisation) ou non stationnaires (résistance de polarisation, diagrammes d'impédance). Nous avons aussi utilisé des comptages de population bactérienne ainsi que des observations et des analyses de surface comme la microscopie électronique à balayage (MEB), la spectroscopie d'électrons pour l'analyse chimique (ESCA), et la spectroscopie à décharge luminescente (SDL), et la micro analyse nucléaire (MAN) qui feront l'objet d'une communication ultérieure.

Le tracé des courbes de polarisation a été effectué à partir du potentiel d'abandon vers les potentiels cathodiques jusqu'à la valeur de $-1000 \mathrm{mV} / \mathrm{ECS}$ suivi d'un "retour» jusqu'à l'obtention de $+1000 \mathrm{mV} / \mathrm{ECS}$ ou d'une densité de courant de $10^{-3} \mathrm{~A} / \mathrm{cm}^{2}$. La vitesse de balayage était de $400 \mathrm{mV} /$ heure.

Nous avons étudié les échantillons après différentes durées d'immersion dans le milieu d'étude, afin de mettre en évidence le paramètre temps dans les processus observés. Les quatre périodes choisies sont les suivantes : éprouvette fraichement polie, et résultats après 1 jour, 1 semaine, ou 1 mois d'immersion.

\section{Étude en eau de mer synthétique}

\section{Milieu sans bactéries}

Cette partie du travail est destinée à servir de base aux travaux qui suivent. En effet, dans ce milieu standard, sans bactéries il est possible de voir l'influence des éléments d'addition sur les caractéristiques électrochimiques. Nous examinerons ensuite l'influence de la présence de bactéries sur les paramètres observés.

L'évolution dans le temps des potentiels d'abandon de chacune des trois nuances pendant un mois conduit à observer (fig. 1) qu'au bout de ce laps de temps, c'est le potentiel de l'acier $316 \mathrm{~L}$ non modifié qui est le plus élevé, malgré une chute de ce paramètre vers une semaine, qui pourrait bien correspondre à une forme de corrosion localisée qui se serait repassivée ensuite.

(*) Les opérations de refusion, de traitement thermique, et de mise en forme ont été réalisées par les laboratoires Unirec d'Usinor-Sacilor à Unieux, grâce à des micro coulées expérimentales.

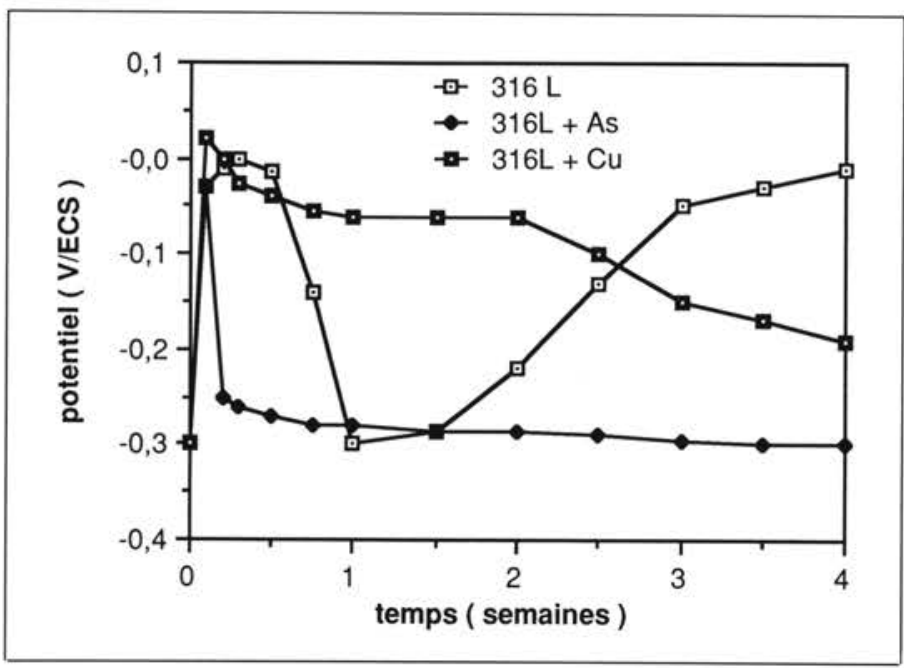

Fig. 1 - Evolution avec le temps des potentiels d'abandon en eau de mer synthétique (ASTM).

Fig. 1 - Rest potential evolution versus the time in synthetic sea water (ASTM).

Les courbes de polarisation obtenues dans le sens « retour » montrent clairement l'influence du temps d'immersion de l'acier $316 \mathrm{~L}$ sur la reconstruction du film passif après le polissage (fig. 2). La passivité semble être obtenue plus rapidement avec le spécimen contenant le cuivre (fig. 3). Pour l'acier à l'arsenic (fig. 4), c'est l'échantillon plongé le plus longtemps qui présente le palier de passivité correspondant au plus faible courant.

Les diagrammes d'impédance font aussi apparaître de fortes variations dans les modules : si au bout d'une journée c'est l'acier à l'arsenic qui présente de très loin le plus grand module d'impédance, la situation est nettement inversée au bout d'une semaine. Cependant, après un mois d'immersion, les trois nuances présentent des caractéristiques voisines, avec une résistance de transfert voisine de $80 \mathrm{~K} \Omega$. A titre de comparaison, les résultats concernant les aciers $316 \mathrm{~L}$ et $316 \mathrm{~L}+$ As sont mentionnés sur les figures 5 et 6 . La mesure de la résistance de polarisation de ces deux derniers aciers (tableau I) a été aussi effectuée.

\section{Milieu ensemencé de bactéries prélevées sur du métal corrodé}

Nous avons comparé dans cette partie de l'étude les aciers $316 \mathrm{~L}$ et $316 \mathrm{~L}+$ As afin d'examiner le rôle de l'arsenic en présence d'une corrosion bactérienne possible. Les bactéries utilisées correspondent

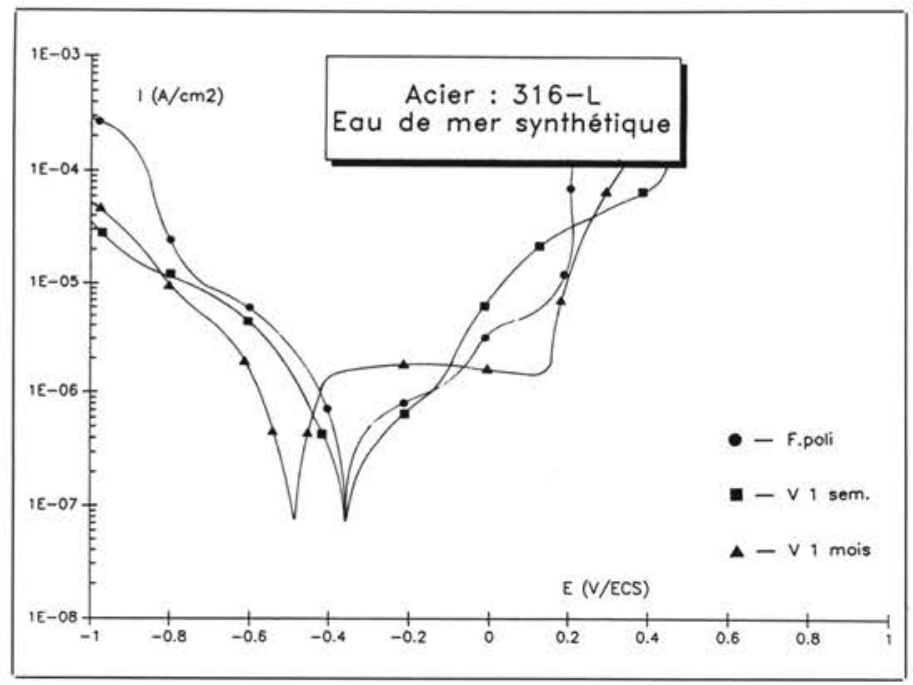

Fig. 2 - Courbe de polarisation de l'acier $316 \mathrm{~L}$ en eau de mer ASTM. Fig. 2 - Polarisation curve of the $316 \mathrm{~L}$ ss in ASTM sea water. 




Fig. 3 - Courbe de polarisation de l'acier $316 \mathrm{~L}+$ As en eau de mer ASTM. Fig. 3 - Polarisation curve of the $316 L+$ As ss ASTM sea water.



Fig. 4 - Courbe de polarisation de l'acier $316 \mathrm{~L}+\mathrm{Cu}$ en eau de mer ASTM. Fig. 4 - Polarisation curve of the $316 L+C u$ ss in ASTM sea water.



Fig. 5 - Diagrammes d'impédance de l'acier $316 \mathrm{~L}$.

Fig. 5 - AC Impedance diagram of the $316 \mathrm{~L}$ ss

$l=A S T M \quad 2=A S T M+$ bact .



Fig. 6 - Diagrammes d'impédance de l'acier $316 \mathrm{~L}+\mathrm{As}$

Fig. 6 - AC Impedance diagram of the $316 L+$ As ss $1=A S T M \quad 2=A S T M+$ bact.

à celles obtenues après ensemencement d'un milieu de culture avec des produits de corrosion prélevés sur un échantillon d'acier ayant séjourné longtemps en eau de mer naturelle. Nous n'avons pas cherché à en déterminer la nature. Nous avons alors inoculé ces bactéries dans le milieu synthétique ASTM.

Si l'on compare les évolutions de potentiel avec le temps, on constate des différences notables de comportement :

- Pour l'acier 316 L non modifié, la présence de bactéries dans le milieu provoque un abaissement des potentiels d'abandon (fig. 7). Ceci correspond à une tendance plus grande de l'alliage à la corrosion généralisée, donc à une moins bonne stabilité du film de passivité.

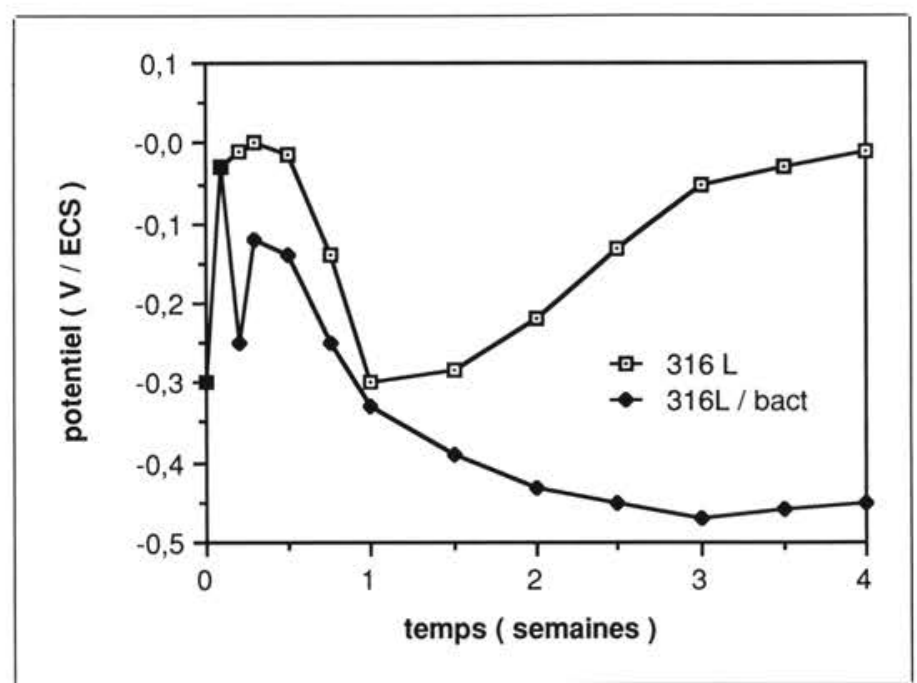

Fig. 7 - Acier $316 \mathrm{~L}$ : Évolution dans le temps des potentiels d'abandon influence des bactéries.

Fig. 7 -316 L ss : Rest potential evolution versus the time; bacteria influence.

- Pour l'acier 316 L à l'arsenic, l'effet est inversé : les potentiels d'abandon ont tendance à croître en présence de bactéries et deviennent beaucoup plus élevés qu'en solution ASTM (fig. 8). Ceci tend à favoriser la sensibilité de l'alliage à la corrosion localisée par piqûres lorsque le potentiel d'abandon se rapproche du potentiel de germination de piqûres.

Ces différences de résultat sont à rapprocher de ceux obtenus par d'autres mesures électrochimiques :

- les diagrammes d'impédance où on constate que la présence de bactéries provoque pour l'acier $316 \mathrm{~L}$ (fig. 5) une diminution la 


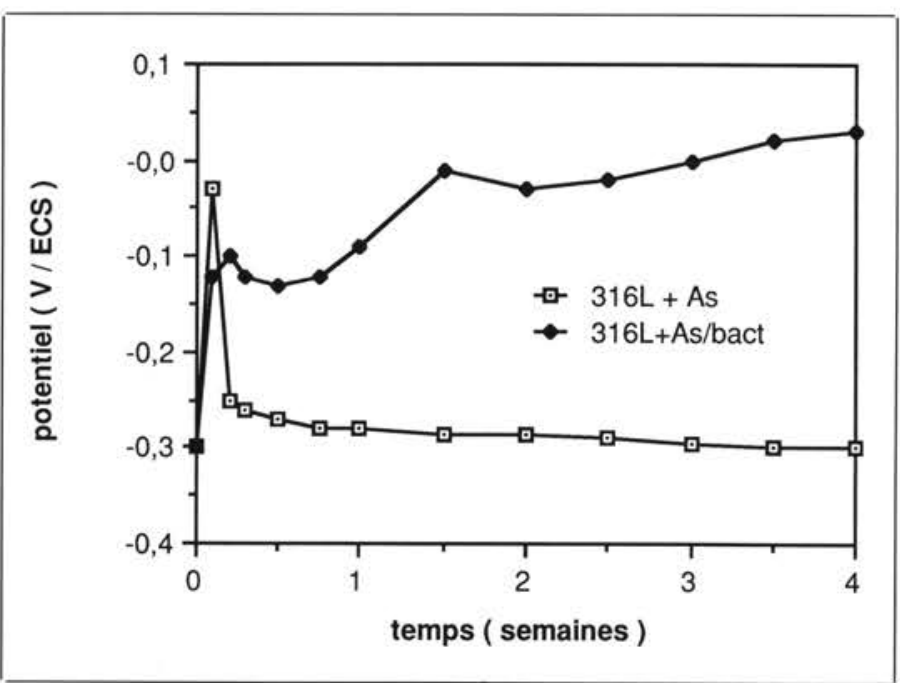

Fig. 8 - Acier $316 \mathrm{~L}+$ As : Évolution dans le temps des potentiels d'abandon influence des bactéries.

Fig. $8-316 L+A s$ ss: Rest potential evolution versus the time; bacteria influence.

résistance de transfert comme la capacité de double couche : l'acier est plus sensible à la corrosion. Pour l'acier $316 \mathrm{~L}+\mathrm{As}$, ces deux paramètres augmentent considérablement dans le milieu inoculé : le film à l'interface possède un caractère protecteur beaucoup plus marqué;

- les résistances de polarisation pour lesquelles les valeurs mesurées permettent de constater des évolutions similaires (tableau I) : Rp diminue en présence de bactéries pour l'acier non modifié et augmente pour l'acier à l'arsenic.

Tableau I - Résistances de polarisation (en $\Omega$ ) mesurées en eau de mer ASTM. Table I - Polarisation resistance (in $\Omega$ ) measurements in synthetic sea water $t$ (immersion $)=1$ mois $(1$ month $)$.

\begin{tabular}{|c|c|c|}
\hline ACIER & SANS BACTÉRIES & AVEC BACTÉRIES \\
\hline $316 \mathrm{~L}$ & 2500000 & 24800 \\
\hline $316 \mathrm{~L}+\mathrm{As}$ & 100000 & 3852000 \\
\hline
\end{tabular}

\section{Étude en eau de mer naturelle}

\section{Eau de mer non traitée}

Pour cette partie du travail, les trois aciers ont été considérés. Nous avons ainsi pu considérer le comportement dans le temps des potentiels d'abandon (fig. 9). Trois phases sont alors mises en évidence :

- durant la première semaine, le potentiel fluctue puis tend vers une valeur plus stable : c'est l'établissement du biofilm. Pour chacune des trois nuances, les valeurs de potentiels restent voisines;

- durant les seconde et troisième semaines, les potentiels sont stables. Celui de la nuance à l'arsenic se fixant à une valeur plus élevée que celle correspondant aux deux autres nuances. Le biofilm durant cette période est stable et ceci peut correspondre, pour les bactéries présentes dans l'eau de mer naturelle, à une période d'incubation;

- durant la quatrième semaine, on constate de brusques changements dans les potentiels. Ceux-ci ont d'abord tendance à croître, puis à décroître. A la fin de ce laps de temps, c'est l'acier qui possède le potentiel le plus élevé, tandis que l'acier à l'arsenic prend un potentiel d'abandon voisin de celui de l'acier non modifié.

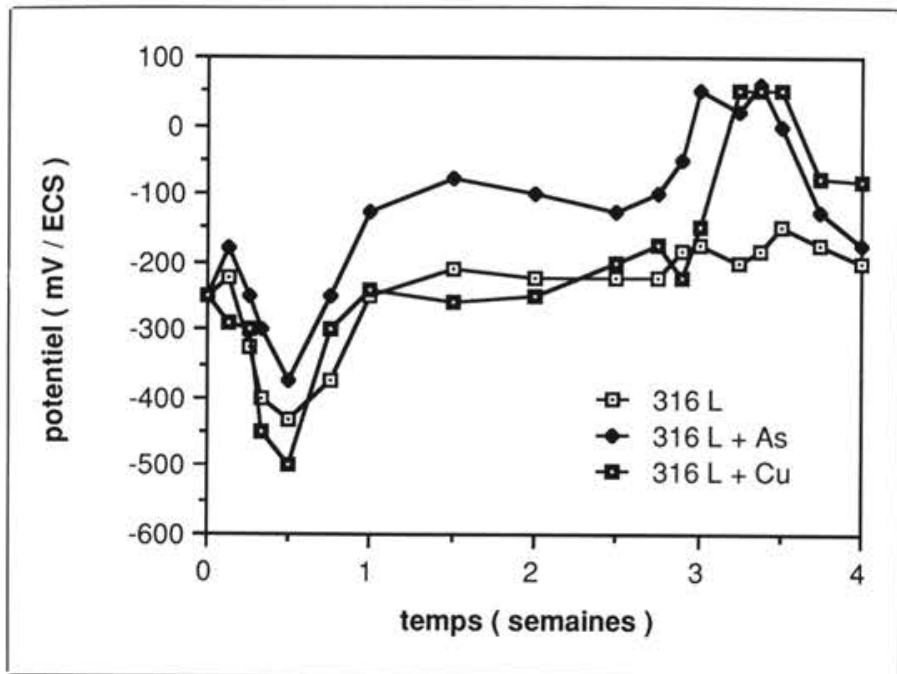

Fig. 9 - Évolution avec le temps des potentiels d'abandon en eau de mer naturelle.

Fig. 9 - Rest potential evolution versus the time in natural sea water.

Les courbes de polarisation montrent que les éléments ajoutés changent le comportement des nuances étudiées, mais ces observations dépendent du temps d'immersion dans le milieu. C'est ainsi que pour l'acier non modifié (fi. 10), les paramètres liés à la passivité (courant, potentiel de piqûre) restent sensiblement constants durant la première semaine, mais ont évolué au bout d'un mois dans le sens d'une déstabilisation du film passif, avec croissance du courant et diminution du potentiel de piqûre. Pour l'acier à l'arsenic (fig. 11), il y a peu de changements de potentiels de piqûre, et le courant de passivité décroît durant la première semaine pour augmenter ensuite et atteindre au bout d'un mois une valeur supérieure à celle du départ. On observe un effet identique nettement plus marqué pour l'acier au cuivre (fig. 12).

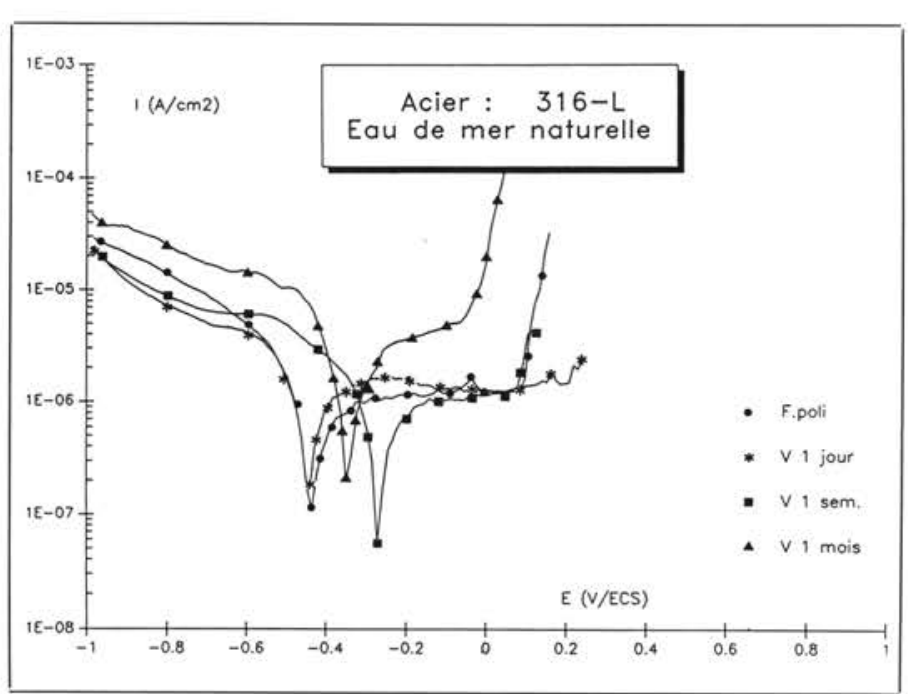

Fig. 10 - Courbe de polarisation de l'acier $316 \mathrm{~L}$ en eau de mer naturelle. Fig. 10 - Polarisation curve of the $316 \mathrm{~L}$ ss in natural sea water.

Les valeurs des résistances de polarisation ont aussi été mesurées, après différents temps d'immersion (tableau II). Les résultats obtenus font apparaître des petites fluctuations, avec une diminution de Rp après un mois, sauf pour le cas de l'acier au cuivre, qui semble dans ce cas présenter une bien meilleure tenue à la corrosion, et $\mathrm{Rp}$ croissant avec le temps d'immersion. 


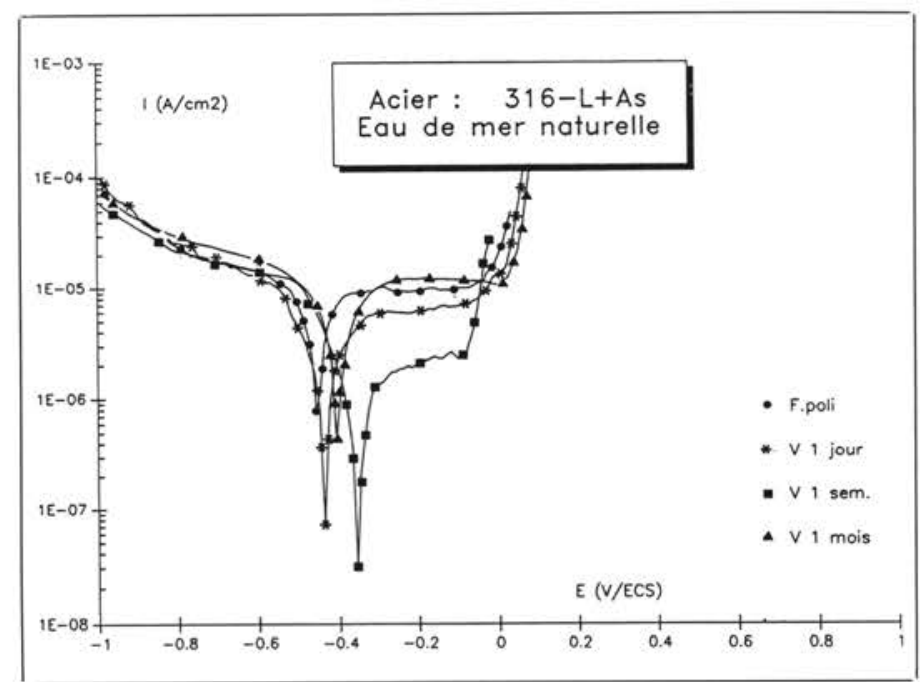

Fig. 11 - Courbe de polarisation de l'acier $316 \mathrm{~L}+$ As en eau de mer naturelle. Fig. 11 - Polarisation curve of the $316 \mathrm{~L}+$ As ss in natural sea water.

Tableau II - Résistances de polarisation (en $\Omega$ ) mesurées en eau de mer naturelle.

Table II - Polarisation resistance (in $\Omega$ ) measurements in natural sea water.

\begin{tabular}{|c|c|c|c|}
\hline $\mathrm{Rp}$ & 1 jour & 1 semaine & 1 mois \\
\hline $316 \mathrm{~L}$ & 230769 & 300000 & 163333 \\
\hline $316 \mathrm{~L}+\mathrm{Cu}$ & 200000 & 727857 & 4045714 \\
\hline $316 \mathrm{~L}+\mathrm{As}$ & 79661 & 111619 & 66470 \\
\hline
\end{tabular}

Ce paramètre temps a aussi une grande influence sur les résultats obtenus à partir des diagrammes d'impédance. Pour l'acier $316 \mathrm{~L}$ non modifié (fig. 13), on obtient une décroissance régulière de la résistance de transfert Rt avec le temps d'immersion. Pour les deux autres nuances, on observe (fig. 14 et 15) que Rt croît entre un jour et une semaine, puis décroît fortement durant les trois semaines suivantes. Les valeurs de Rt déterminées à partir de ces diagrammes sont rassemblées dans le tableau III.



Fig. 12 - Courbe de polarisation de l'acier $316 \mathrm{~L}+\mathrm{Cu}$ en eau de mer naturelle. Fig. 12 - Polarisation curve of the $316 L+C u$ ss in natural sea water.

Tableau III - Résistances de transfert (en K $\Omega$ ) après immersion des aciers étudiés.

Table III - Transfert resistances (in KS) after immersion of studied steels.

\begin{tabular}{|c|c|c|c|}
\hline Rt & 1 jour & 1 sem. & 1 mois \\
\hline $316 \mathrm{~L}$ & 430 & 294 & 29 \\
\hline $316 \mathrm{~L}+\mathrm{Cu}$ & 148 & 383 & 25 \\
\hline $316 \mathrm{~L}+\mathrm{As}$ & 75 & 375 & 13 \\
\hline
\end{tabular}

Le dénombrement de la flore bactérienne totale après ces différents temps d'immersion ne montre pas de différences notables, au bout d'un mois, quant au nombre total de bactéries présentes à la surface de ces trois aciers. Il est proche, dans les trois cas, de $1,510^{6}$ bactéries $/ \mathrm{cm}^{2}$. Le décompte des bactéries viables hétérotrophes montre (tableau IV) que la présence d'éléments d'alliages aurait tendance à plutôt favoriser la croissance de bactéries aérobies, sans favoriser celle des bactéries anaérobies, dont la quantité reste voisine de zéro au bout d'un mois d'immersion.

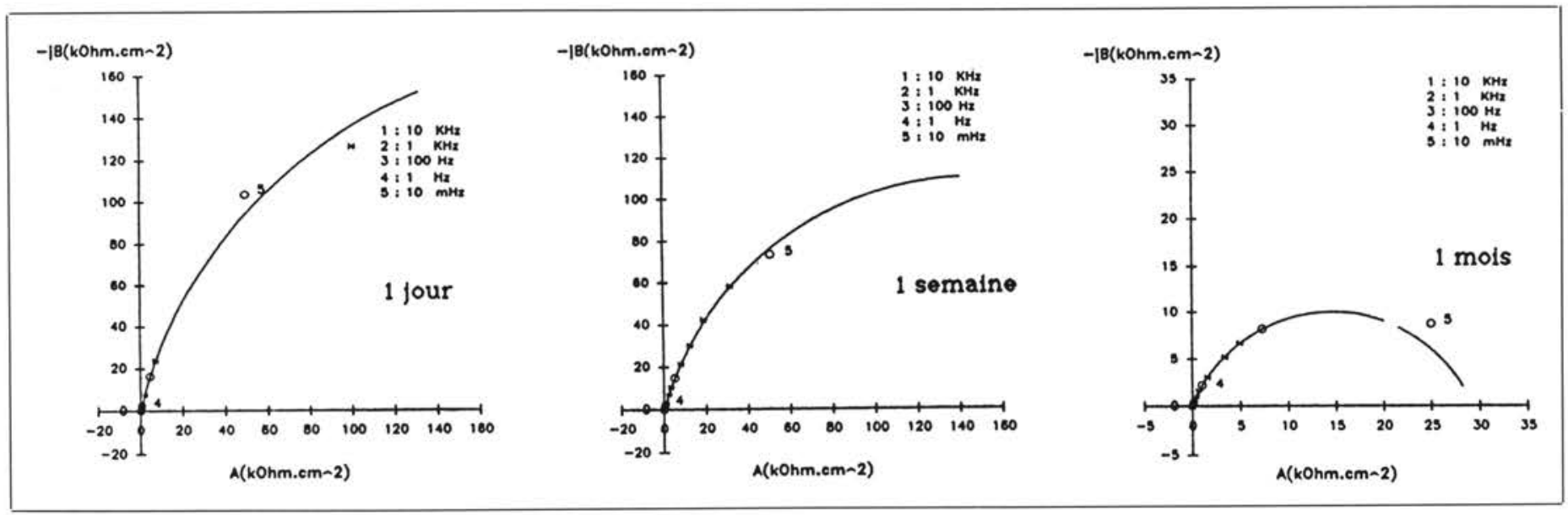

Fig. 13 - Diagrammes d'impédance de l'acier $316 \mathrm{~L}$ après différents temps d'immersion.

Fig. 13 - Impedance diagrams of the $316 \mathrm{~L}$ ss after different immersion times. 


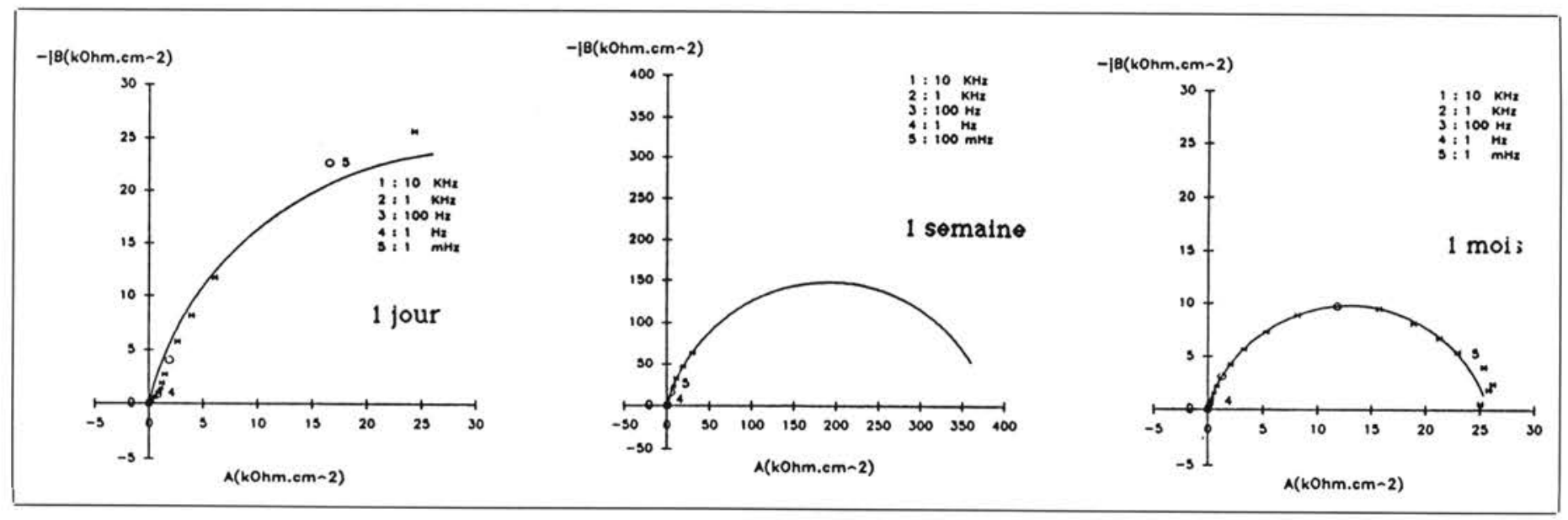

Fig. 14 - Diagrammes d'impédance de l'acier $316 \mathrm{~L}+\mathrm{Cu}$ après différents temps d'immersion.

Fig. 14 - Impedance diagrams of the $316 \mathrm{~L}+\mathrm{Cu}$ ss after different immersion times.

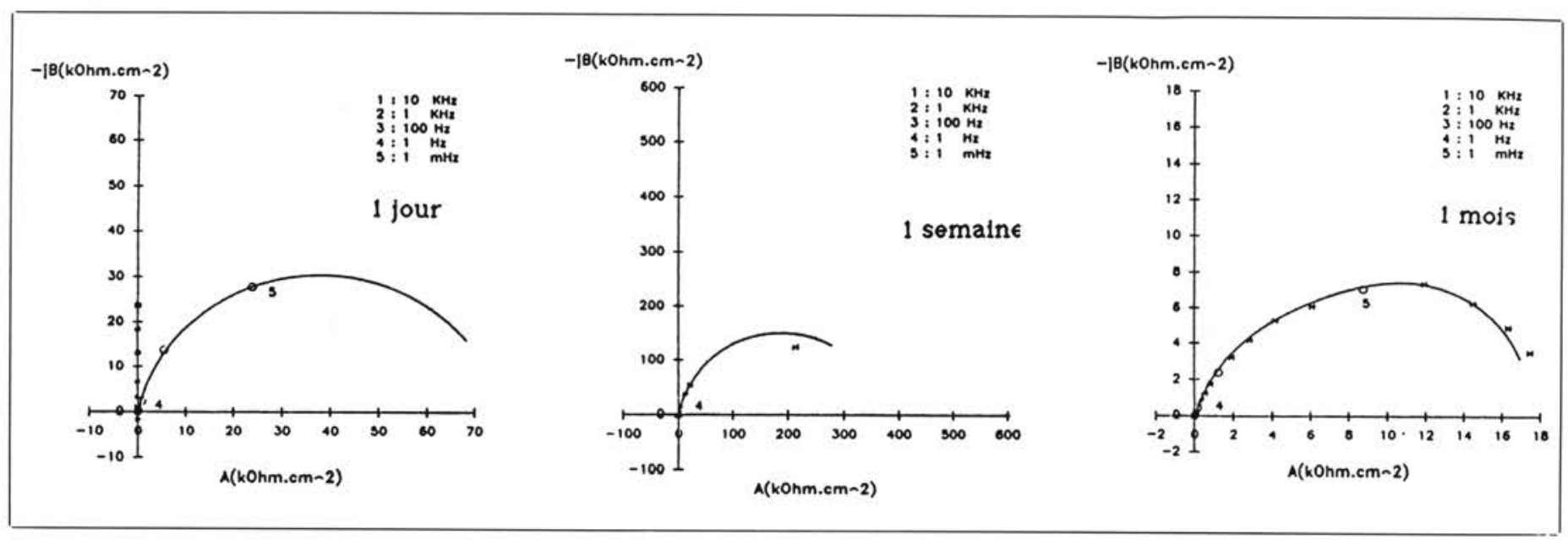

Fig. 15 - Diagrammes d'impedance de l'acier $316 \mathrm{~L}+$ As après différents temps d'immersion.

Fig. 15 - Impedance diagrams of the $316 \mathrm{~L}+$ As ss after different immersion times.

Tableau IV - Nombre de bactéries viables à la surface des aciers.

Table IV - Number of viable bacteria on the steels surface.

\begin{tabular}{|c|cc|cc|}
\hline \multirow{2}{*}{$\begin{array}{c}\text { N Viables } \\
\text { Heterotr. }\end{array}$} & \multicolumn{2}{|c|}{ 1 SEMAINE } & \multicolumn{2}{c|}{1 MOIS } \\
\cline { 2 - 5 } & Aero. & Anaero. & Aero. & Anaero. \\
\hline $316 \mathrm{~L}$ & $8.2 . \mathrm{E} 3$ & 0 & $2.2 . \mathrm{E} 3$ & 0 \\
\hline $316 \mathrm{~L}+\mathrm{Cu}$ & $6.6 . \mathrm{E} 3$ & 0 & $4.3 . \mathrm{E} 3$ & 0 \\
\hline $316 \mathrm{~L}+\mathrm{As}$ & $7.3 . \mathrm{E} 3$ & $1.4 . \mathrm{E} 2$ & $6.2 . \mathrm{E} 3$ & 0 \\
\hline
\end{tabular}

\section{Eau de mer stérilisée puis ensemencée}

Afin de mieux contrôler la nature de la flore bactérienne dans le milieu d'étude, nous avons utilisé l'eau de mer naturelle "vivante» précédente, mais tout d'abord stérilisée, puis ensuite ensemencée à l'aide d'un milieu de culture, pour être enfin inoculée de bactéries choisies. Celles-ci sont au nombre de deux, et nous les avons utilisées successivement au cours du même essai : dans un premier temps, le milieu ne contenait que la souche "vibrio natrigens" (VN), aérobie, puis au bout de trois semaines, était inoculée la souche «Desulfovibrio vulgaris" (DV), anaérobie facultative. Cette séquence avait pour but de tenter de reproduire à l'aide de ces deux seules bactéries la croissance initiale du voile biologique au dépend du film passif. En effet, on aurait au départ la bactérie aérobie qui consommerait l'oxygène de ce film, puis ensuite le milieu appauvri en oxygène deviendrait favorable à la prolifération d'une souche anaérobie.
Nous avons utilisé les mêmes techniques d'étude que dans les cas précédents, pour comparer les résultats obtenus dans des cas similaires, notamment lors de l'emploi de l'eau de mer naturelle. Les essais ont toutefois été prolongés car il nous a semblé intéressant d'observer les évolutions jusqu'à trois semaines après la seconde incubation.

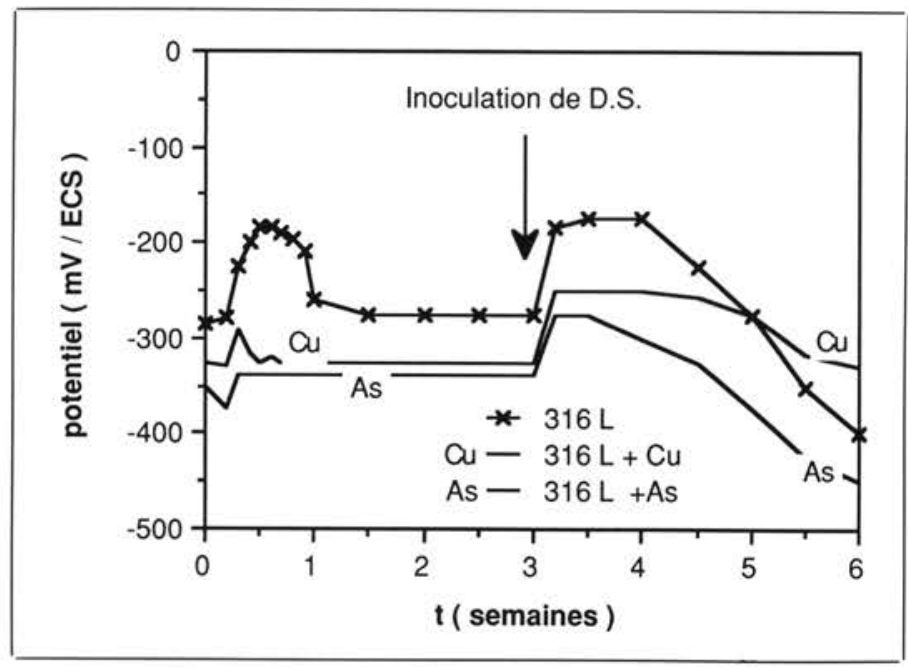

Fig. 16 - Evolution avec le temps des potentiels d'abandon en eau de mer stérilisée inoculée.

Fig. 16 - Rest potential evolution versus the time in sterilized and inoculated sea water. 


\section{PRÉVENTION DE LA BIOCORROSION PAR MODIFICATION DE LA COMPOSITION CHIMIQUE}

La figure 16 montre par exemple les évolutions de potentiels durant les six semaines de tests pour les trois nuances étudiées. Le saut de potentiel obtenu au bout de trois semaines est systématique, et nous l'avons attribué à la technique de l'inoculation elle-même. On remarque bien là aussi une stabilisation du potentiel d'abandon pendant au moins les seconde et troisième semaines, correspondant à une période de stabilité du biofilm formé durant le début des mesures. On constate sur cette figure qu'en présence de bactéries desulfovibrio vulgaris, les potentiels diminuent, mais que pour l'alliage contenant du cuivre, cette décroissance est moins rapide. A la fin de la mesure, l'acier non modifié et l'acier à l'arsenic tendent vers le même potentiel, plus bas que celui de l'acier au cuivre.

Les courbes de polarisation ont été effectuées au bout de trois semaines (bactérie VN) et de six semaines (bactéries VN + DS), et pour les trois nuances. Les résultats obtenus montrent que :

- pour l'acier $316 \mathrm{~L}$, le courant de passivité est plus élevé au bout des six semaines qu'à la fin des trois premières semaines (fig. 17);

- pour l'acier $316 \mathrm{~L}+$ As, ce courant est peu modifié (fig. 18);

- pour l'acier $316 \mathrm{~L}+\mathrm{Cu}$, ce courant a nettement diminué (fig. 19).

Dans chacun des cas, le potentiel de piqûre est peu affecté.

Des diagrammes d'impédances ont aussi été tracés durant ces essais. à chaque semaine. De grandes variations dans les valeurs des

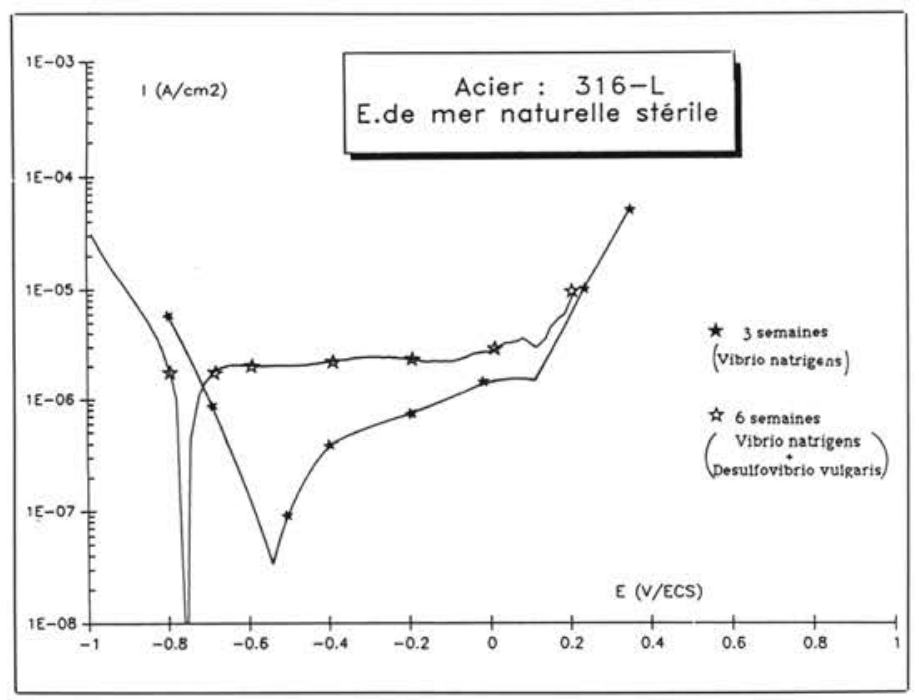

Fig. 17 - Courbe de polarisation de l'acier 316L en eau de mer stérilisée inoculée.

Fig. 17 - Polarisation curve of the 316 L ss in sterilized inoculated sea water.

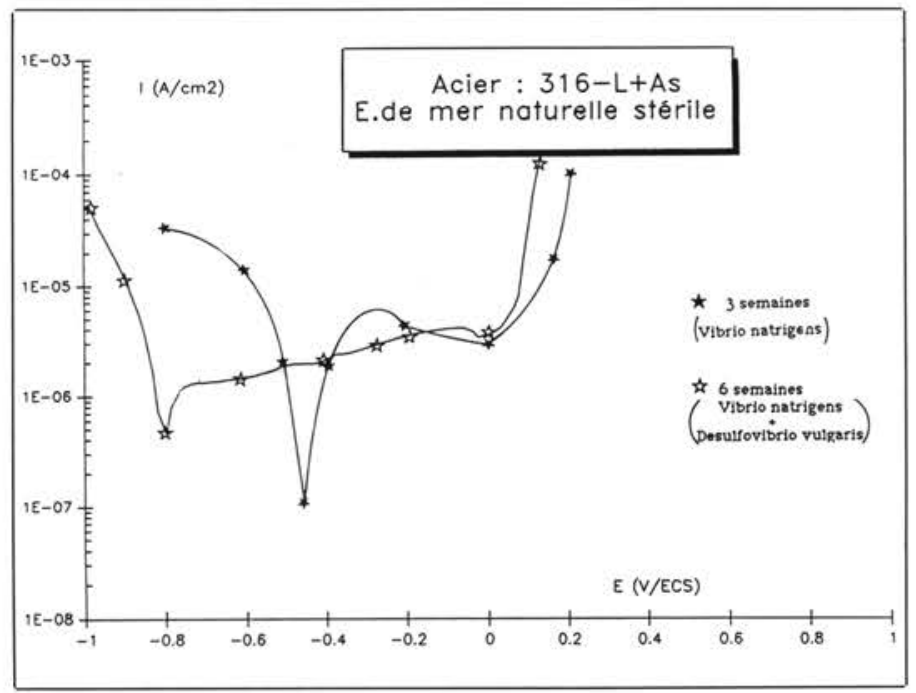

Fig. 18 - Courbe de polarisation de l'acier $316 \mathrm{~L}+$ As en eau de mer modifiée. Fig. 18 - Polarisation curve of the $316 L+$ As ss in modified sea water. résistances de transfert peuvent alors être observées. La détermination de ces valeurs conduit à l'établissement du tableau V. On remarque là encore que l'acier au cuivre possède un comportement différent des deux autres nuances : a partir du moment ou le biofilm se stabilise, les valeurs de Rt sont constamment croissantes, manifestant une sensibilité de moins en moins grande à la corrosion. Pour l'acier non modifié et pour l'acier à l'arsenic, l'inoculation de la bactérie désulfovibrio vulgaris semble accélérer au moins pour un temps la vitesse de corrosion, puisqu'on met alors en évidence une décroissance de Rt, non totalement compensée par la suite.

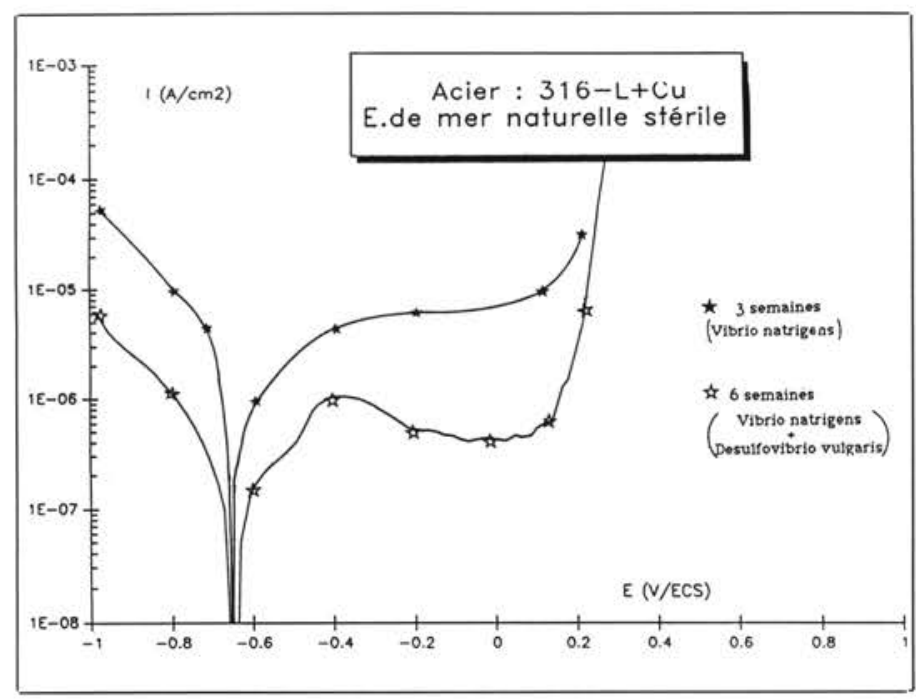

Fig. 19 - Courbe de polarisation de l'acier $316 \mathrm{~L}+$ Cu en eau de mer modifiée. Fig. 19 - Polarisation curve of the $316 \mathrm{~L}$ ss in modified sea water.

Tableau $\mathbf{V}$ - Résistances de transfert (en $\mathrm{K} \Omega$ ) après immersion des aciers étudiés en eau de mer modifiée.

Table $V$ - Transfer resistances (in $K \Omega$ ) after immersion of studied steels in modified sea water.

\begin{tabular}{|c|c|c|c|c|c|}
\hline \multirow{2}{*}{ Rt } & \multicolumn{2}{|c|}{ VIBRIO NATRIGENS } & \multicolumn{2}{c|}{ VN + DV } \\
\cline { 2 - 6 } & 1 jour & 1 sem. & 3 sem. & 4 sem. & 6 sem. \\
\hline $316 \mathrm{~L}$ & 172 & 758 & 903 & 683 & 767 \\
\hline $316 \mathrm{~L}+\mathrm{Cu}$ & 128 & 61 & 314 & 331 & 978 \\
\hline $316 \mathrm{~L}+\mathrm{As}$ & 156 & 462 & 640 & 22 & 582 \\
\hline
\end{tabular}

\section{Discussion des résultats}

\section{Effet du vieillissement}

Il apparaît tout d'abord à l'examen de ces résultats d'essais électrochimiques, comme à la suite de dénombrements microbiologiques, que le comportement de chacun des matériaux étudiés, et dans chaque milieu considéré, synthétique ou naturel, ensemencé ou non, que le temps de l'immersion joue un rôle considérable sur le comportement du matériau. C'est ainsi que le vieillissement du biofilm présent à l'interface met en évidence trois périodes: la première semaine correspond à la formation de ce biofilm, les deux suivantes environ à sa stabilité, et à partir de la quatrième à sa modification.

En effet, durant la première phase, l'existence du film passif des aciers inoxydables conduit à une présence importante d'oxygène à l'interface solide-liquide : ceci favorise la croissance de bactéries aérobies qui vont provoquer la formation du biofilm. Les souches commencent alors à s'installer, puis à coloniser la surface en consommant l'oxygène du film passif, qui se détruit progressivement. 


\section{LEMAITRE, G. HERNANDEZ, G. BERANGER, J. GUEZENNEC}

Le biofilm qui se forme proviendrait des produits du métabolisme de ces bactéries (par polymérisation de polysaccharides par exemple) : c'est la seconde période. Lorsque l'oxygène est en voie de disparition, le milieu est localement désaéré près de l'interface, et les bactéries anaérobies peuvent alors se multiplier. Le métabolisme de ces bactéries étant différent, les produits obtenus ne sont plus les mêmes : très souvent, on peut avoir formation de produits soufrés qui diminuent fortement la tenue à la corrosion.

Le rôle recherché pour les éléments ajoutés à la composition de l'acier inoxydable serait alors de combattre au moins l'une de ces étapes et de freiner ainsi soit la destruction du film passif par les bactéries aérobies, soit la formation de produit «indésirables» dûs au métabolisme des bactéries anaérobies.

\section{Influence de l'arsenic}

En milieu synthétique, les potentiels d'abandon évoluent avec le temps d'immersion, et il apparaît que la présence de bactéries dans le milieu inverse les évolutions. On constate en effet que pour l'acier sans arsenic la présence de bactéries conduit à favoriser la corrosion généralisée par un abaissement des potentiels d'abandon, donc par une destabilisation générale du fil passif. Par contre, pour l'acier modifié par la présence d'arsenic, c'est une élévation des potentiels qui est observable dans ces mêmes conditions. Mais le potentiel d'abandon atteint alors des valeurs voisines de $0 \mathrm{mV} / \mathrm{ECS}$, comme l'indique la figure 8 . Si on compare avec les indications fournies par les courbes de polarisation de cet acier (fig. 3), on remarque que cette valeur de potentiel correspond à un domaine où la probabilité de formation de piqûres est très importante. Par conséquent, la présence de ces mêmes bactéries conduit, pour l'acier à l'arsenic, à favoriser la corrosion par piqûres due à une élévation des potentiels d'abandon, donc par destabilisation localisée du film passif.

Ces observations peuvent expliquer les résultats obtenus à l'aide des autres techniques électrochimiques: pour l'acier $316 \mathrm{~L}$ les diagrammes d'impédance montrent une forte accélération de la vitesse de corrosion en présence de bactéries, tandis que pour l'acier modifié à l'arsenic, on remarque une évolution inverse, qui correspond alors à une diminution de la corrosion généralisée.

En milieu naturel, on remarque là aussi une élévation des potentiels d'abandon avec la modification de la composition (fig. 9). C'est surtout durant la seconde période que l'alliage s'anoblit, avec un potentiel voisin de $-100 \mathrm{mV} / \mathrm{ECS}$, où le risque de germination de piqûres est moins important. Toutefois, au bout d'un mois, les potentiels des nuances $316 \mathrm{~L}$ et $316 \mathrm{~L}+$ As tendent à se regrouper, après que celui de l'acier modifié soit passé au bout de trois semaines par des potentiels proches de $+50 \mathrm{mV} / \mathrm{ECS}$, c'est-à-dire que la corrosion localisée s'est probablement amorcée. Il est alors logique de constater que, pour cette nuance, le courant de passivité a augmenté après un mois de vieillissement (fig. 11) alors qu'il avait tendance à diminuer avec le temps d'immersion durant la première semaine. Il en est de même pour les valeurs de résistances de polarisation comme de transfert qui augmentent au bout d'une semaine, puis diminuent après un mois d'immersion.

En eau de mer stérilisée puis ensemencée, on remarque que l'addition d'arsenic dans la composition de l'acier modifie très peu la courbe de polarisation obtenue au bout de trois semaines (en présence de Vibrio Natrigens seule). Par contre, à la fin de l'essai (en présence des deux bactéries), la comparaison des deux courbes obtenues à l'aide des deux nuances montre essentiellement une forte augmentation du courant de passivité lorsque l'arsenic est ajouté.

L'ensemble de ces observations conduit à ne pas attribuer un rôle positif à l'arsenic comme élément d'addition pour améliorer la résistance à la biocorrosion des aciers inoxydables.

\section{Action du cuivre}

La figure 1 montre que l'addition de cuivre à l'acier $316 \mathrm{~L}$ conduit à abaisser le potentiel d'abandon en milieu synthétique ASTM au bout d'un mois, mais plus faiblement que pour l'addition d'arsenic. Les risques de corrosion par piqûres semblent alors être évités, et les risques de corrosion généralisée minimisés. On constate en effet sur la courbe de polarisation de la figure 8 qu'un palier de passivité se manifeste et que le courant de passivité tend à diminuer avec le temps d'immersion. Ces observations sont des résultats encourageants quant à la tenue de l'alliage au cuivre en milieu marin. Mais qu'en est-il en présence de bactéries?

En milieu naturel, l'évolution du potentiel d'abandon avec le temps (fig. 9) suit une courbe similaire à celle de l'acier à l'arsenic. Toutefois, on peut faire deux remarques :

- Le potentiel reste identique à celui de l'acier non modifié durant les trois premières semaines.

- Après ce laps de temps, le potentiel augmente brusquement jusqu'à $+50 \mathrm{mV} / \mathrm{ECS}$, mais ne redescend pas vers celui de l'acier non modifié. A la fin de la mesure, il se situe vers $-100 \mathrm{mV} / \mathrm{ECS}$. $\mathrm{Si}$ on compare ces potentiels aux courbes de polarisations correspondantes (fig. 12), on constate que des risques de corrosion localisée existent à $+50 \mathrm{mV} / \mathrm{ECS}$ mais que $-100 \mathrm{mV} / \mathrm{ECS}$ correspondrait à une passivité stable. On constate malgré tout que le courant de passivité diminue durant la première semaine, mais qu'il a considérablement augmenté au bout d'un mois. Ceci tend à confirmer que la corrosion localisée a pu se produire.

Cette constatation va dans le même sens que les résultats obtenus par la détermination des résistances de transfert : alors qu'avec l'acier non modifié, on a une décroissance régulière de $\mathrm{Rt}$, avec l'acier au cuivre il y a d'abord une croissance, puis une diminution.

En eau de mer stérilisée ensemencée, c'est surtout après la seconde inoculation que l'on constate des différences de comportement intéressantes (fig. 16). Si, après six semaines d'essai, l'acier non modifié et l'acier à l'arsenic ont des potentiels voisins, l'acier au cuivre possède un potentiel plus élevé et qui décroît moins vite : il devrait donc résister plus longtemps à la corrosion généralisée. C'est bien ce que semblent indiquer les mesures de Rt (tableau V). L'amélioration de la situation se fait surtout sentir à partir de la seconde inoculation, comme si le cuivre réagissait en contrecarrant les effets négatifs dûs au métabolisme de Désulfovibrio Vulgaris. Ceci est d'ailleurs confirmé par la courbe de polarisation de la figure 18 .

On constate donc que l'addition de cuivre à l'acier $316 \mathrm{~L}$ tend à améliorer la tenue de l'alliage à la biocorrosion. Cependant, les risques de corrosion par piqûres ne sont pas à négliger.

\section{Conclusions}

Des mesures électrochimiques et microbiologiques ont été pratiquées sur trois nuances d'acier $316 \mathrm{~L}$, l'une non modifiée et les deux autres auxquelles ont été ajoutés à l'état liquide de l'arsenic ou du cuivre. Quatre milieux ont été utilisés pour étudier la résistance à la corrosion de ces nuances :

- une eau de mer synthétique préconisée par l'ASTM

- la même solution ensemencée et inoculée

- une eau de mer naturelle

- la même eau de mer, mais stérilisée, puis ensemencée et inoculée.
Ces différentes conditions ont permis de comparer les milieux entre eux, mais surtout de voir l'influence de la présence de l'élément ajouté à l'alliage par une comparaison du comportement de chaque alliage. Il ressort de cette étude les points suivants :

- L'eau de mer synthétique reconstituée en laboratoire n'est pas un milieu susceptible de reproduire le comportement de l'alliage à la biocorrosion. Les résultats obtenus dans chaque solution sont en effet très différents. 


\section{PRÉVENTION DE LA BIOCORROSION PAR MODIFICATION DE LA COMPOSITION CHIMIQUE}

- Trois périodes ont pu être mises en évidence, correspondant à trois étapes du processus : la formation du biofilm, sa stabilité, puis son évolution. Ce ne sont pas forcément les mêmes bactéries qui agissent à chaque étape, car les concentrations locales en oxygène à l'interface évoluent avec le temps.

- L'arsenic est un élément d'addition qui a tendance à déstabiliser le film passif des aciers inoxydables. Même si le biofilm qui se forme en présence de bactéries semble conférer une certaine protection à la surface, les conditions deviennent alors telles que c'est la corrosion par piqûres qui est favorisée. Cet élément n'est pas indiqué pour combattre la biocorrosion.

- Le cuivre confère à l'interface de meilleures caractéristiques de tenue à la corrosion que l'arsenic. Cet élément semble être le plus efficace lors de la troisième phase, c'est-à-dire lorsque la solution à l'interface est désoxygénée : c'est la période de croissance des bactéries anaérobies. Un risque de formation de piqûres a pu être décelé, mais cela peut provenir de la méthode d'inoculation ellemême qui a fait augmenter d'une façon systématique tous les potentiels d'abandon au cours de la mesure. Des études sur des périodes de temps plus longues devraient pouvoir donner une idée plus nette des phénomènes mis en jeu.

\section{Remerciements}

Ces travaux n'auraient pas pu être entrepris sans la collaboration et le soutien des laboratoires d'Usinor Sacilor (Unirec) à Unieux (Loire) ainsi que d'Ifremer, centre de Brest (Finistère). Ils ont été financièrement soutenus par le Ministère de la Recherche et de la Technologie (Décision d'aide $\mathrm{n}^{\circ} 85 \mathrm{~S} 238$ ), dans le cadre d'une recherche commune entre les trois laboratoires.
[1] W. A. Hamilton, Ann. Rev. Microbiol., 39 (1985), 195-217.

[2] S. Kueelleberg, B. A. Humphrey, K. C. Marshall, App. Environ. Microbiol., 43 (1982), 1161-1172.

[3] G. H. Bоoth, A. K. Tiller, Corr. Sci. 8 (1968), 583-600.

[4] M. N. McDonald, D. D. McDonald, J. Electrochem. Soc. 134,1 (1987), $41-46$.
[5] G. OKamoto, T. Shibata, « Passivity of metals ", Corosion Monograph Series, R. P. Frankenthal and J. Kruger Ed., Electrochem. Soc., Peddington, New Jersey (1978), p. 646.

[6] J. G. StOECKeR, Materials Performance, (1984), 48-55.

[7] R. JOHSEN, E. BARDAL, Corrosion, 5 (1985), 269-302.

\section{Céramique}

\section{ROULEMENT CÉRAMIQUE : EMPLOI EN CONDITIONS EXTREMES}

L'évolution technologique impose aux roulements de fonctionner avec des charges et à des vitesses toujours plus élevées tout en satisfaisant à des exigences d'encombrement et d'environnement toujours plus sévères. Pour pallier les limitations d'emplois des aciers alliés et des lubrifiants classiques dans certaines applications, la Société SNR a étudié des roulements en céramique.

\section{Roulement en nitrure de silicium}

La céramique à roulement est par excellence le nitrure de silicium en raison de sa faible densité, de la grande dureté, de sa résistance à l'usure et à la fatigue, de sa tenue aux températures élevées et de sa grande stabilité thermique. Ses propriétés d'amagnétisme et sa résistivité électrique sont utilisées en outre pour des emplois définis.

Les caractéristiques mécaniques du nitrure de silicium étant très différentes de celles de l'acier, SNR d'une part a été amené à redéfinir la géométrie interne du roulement, et, d'autre part, a dû mettre en œuvre de nouvelles techniques de rectification, notamment pour la finition des billes de haute précision et celle des pistes de roulement.

Les données géométriques, mécaniques et cinématiques de l'ensemble, ont été prises en considération dans le logiciel de calcul SNR - Softroll pour définir la microgéométrie des surfaces en contact.

Les travaux sur le comportement des contacts sous lubrification partielle ont entrainé de nouvelles règles de conception assurant une plus grande tolérance au roulement.

Par ailleurs, l'inertie chimique de la céramique comparée à l'acier a pour conséquence une affinité plus faible entre les surfaces en contact. Les phénomènes de grippage sont ainsi retardés et le roulement peut même fonctionner un temps en l'absence de lubrification.

\section{Applications}

L'emploi de la céramique dans les roulements a étendu non seulement leurs durées de vie dans des conditions difficiles mais aussi élargi leur domaine d'emploi.
Les roulements hybrides à bagues en acier et billes en céramique sont utilisés pour les machines à grandes vitesses de rotation : turbo-machines, machines-outils, gyroscopes. La réduction de la force centrifuge due à la faible densité des billes permet d'accrồtre sensiblement la vitesse limite de roulement. Et le module d'élasticité élévé augmente la rigidité des roulements, caractéristique fondamentale des machines-outils de précision.

Pour des applications particulières (industrie du vide, moteur électrique à haute température, ...) certains roulements ont des bagues en métal ayant des caractéristiques définies, entraînant une meilleure résistance au grippage, accrue encore par l'emploi de lubrifiants spéciaux : graisses pour hautes températures, à faible taux d'évaporation, solides.

Les roulements tout céramique sont choisis pour leur inertie chimique et employés dans les industries pétrolière, chimique, nucléaire, cryogénique, ...

Le roulement est apte à fonctionner dans divers fluides agressifs pour le métal. L'emploi de joints sophistiqués de protection n'est plus nécessaire. 